\title{
Prospective multicenter Polish Stress Echocardiography Registry (PolStress-Echopro) - the role in clinical practice
}

\author{
Zbigniew Tadeusz Gąsior 1,2,A,C-F, Bartosz Lasota ${ }^{2, B, D-F}$, Beata Zaborska ${ }^{3, C, E, F}$, Katarzyna Mizia-Stec ${ }^{4, B, C, E, F}$, \\ Piotr Gościniak ${ }^{5, B, C, E, F}$, Marta Marcinkiewicz-Siemion ${ }^{6, B, C, E, F}$, Barbara Brzezińska, ${ }^{7, B, E, F}$, Jolanta Rapacewicz ${ }^{8, B, E, F}$, \\ Jolanta Rzucidło-Resil ${ }^{9, B, E, F}$, Tomasz Gąsior ${ }^{10, B, D-F}$, Agnieszka Olszanecka ${ }^{11, B, E, F}$, Edyta Płońska-Gościniak $8, A-C, E, F$ \\ ${ }^{1}$ Department of Cardiology, School of Health Sciences in Katowice, Medical University of Silesia, Poland \\ ${ }^{2} 2^{\text {nd }}$ Department of Cardiology Upper Silesian Medical Centre, Katowice, Poland \\ ${ }^{3}$ Department of Cardiology, Postgraduate Medical School, Grochowski Hospital, Warszawa, Poland \\ ${ }^{4} 1^{\text {st }}$ Department of Cardiology, School of Health Sciences in Katowice, Medical University of Silesia, Poland \\ ${ }^{5}$ Department of Cardiology, Provincial Hospital, Szczecin, Poland \\ ${ }^{6}$ Department of Cardiology, University Hospital, Bialystok, Poland \\ ${ }^{7}$ Department of Cardiology, T. Marciniak Hospital, Wrocław, Poland \\ ${ }^{8}$ Department of Cardiology, Pomeranian Medical University in Szczecin, Poland \\ ${ }^{9}$ Department of Coronary Artery Disease and Heart Failure, John Paul II Hospital, Kraków, Poland \\ $103^{\text {rd }}$ Department of Cardiology and Structural Heart Diseases, School of Medicine in Katowice, Medical University of Silesia, Poland \\ ${ }^{11} 1^{\text {st }}$ Department of Cardiology, Interventional Electrocardiology and Hypertension, Jagiellonian University Medical College, Kraków, Poland \\ A - research concept and design; B - collection and/or assembly of data; C - data analysis and interpretation; \\ $D$ - writing the article; $E$ - critical revision of the article; $F$ - final approval of the article
}

Address for correspondence

Zbigniew Gąsior

E-mail:zbgasior@gmail.com

Funding sources

None declared

Conflict of interest

None declared

Received on July 27, 2018

Reviewed on October 24, 2018

Accepted on December 11, 2018

Published online on November 19, 2019

Cite as

Gąsior ZT, Lasota B, Zaborska B, et al. Prospective multicenter Polish Stress Echocardiography Registry (PolStressEchopro) - the role in clinical practice. Adv Clin Exp Med. 2019;28(11):1555-1560. doi:10.17219/acem/100648

DOI

10.17219/acem/100648

Copyright

Copyright by Author(s)

This is an article distributed under the terms of the Creative Commons Attribution Non-Commercial License (http://creativecommons.org/licenses/by-nc-nd/4.0/)

\section{Abstract}

Background. Stress echocardiography (SE) is becoming an increasingly frequently performed diagnostic examination in Poland. After the published retrospective PoISTRESS Registry, this prospective study is the first one available so far.

Objectives. The aim of the study was to analyze SE tests, taking into account the clinical characteristics of the patients, indications, applied protocols, and diagnostic and therapeutic decisions.

Material and methods. Reference cardiological centers in Poland were asked for a 1-month prospective analysis of the data obtained. The study included $189 \mathrm{SE}$ examinations. To evaluate coronary artery disease (CAD) (178 tests), all 17 centers performed dobutamine SE (DSE) (100\%), 3 centers (17\%) performed pacing, while cycle ergometer and treadmill SE were performed by $1(5 \%)$ and $2(11 \%)$ centers, respectively. In patients with valvular heart disease (VHD) (11 tests), 3 centers (16\%) performed SE to evaluate low-flow/ low-gradient aortic stenosis (AS), 4 (22\%) in asymptomatic AS and 1 (5\%) to evaluate mitral regurgitation.

Results. For CAD assessment, a positive result was found in 37 (20\%) patients, negative in 109 (61\%) and nondiagnostic in 32 (19\%). In the CAD group, coronarography was performed in 41 (23\%) people. The analysis of the significance of the SE results for decision-making on interventional measures revealed that 30 patients (from the total study population of 189) were referred for the intervention.

Conclusions. The most commonly used SE is the DSE. Negative test results allowed in almost half of the patients to resign from invasive coronarography. Stress echocardiography should be more frequently used in patients with VHD in the qualification for invasive treatment.

Key words: coronary artery disease, stress echocardiography, valvular heart disease 


\section{Introduction}

Stress echocardiography (SE) is a widely known method for the diagnosis of coronary artery disease (CAD) and valvular heart disease (VHD), among others, which involves the use of various types of functional tests stimulating contractility and/or inducing ischemia together with echocardiographic evaluation of contractility and/ or vascular evaluation of valve flows. ${ }^{1}$ In CAD diagnosis, SE, depending on the type of load, is characterized by a high sensitivity and specificity of the test: exercise SE - 80-85\% and $80-88 \%$ respectively, dobutamine SE (DSE) - 79-83\% and $82-86 \%$, SE with vasodilatatory agent - 72-79\% and $92-95 \% .^{2}$ Stress echocardiography is recommended as an initial test to diagnose stable CAD in patients with a pre-test probability of $66-85 \%$ (intermediate) or a left ventricular ejection fraction (LVEF) below 50\% in patients without typical symptomatology. In addition, it is performed in patients with abnormalities in resting electrocardiograpgy (ECG), which prevent accurate interpretation of ECG changes during loading, in symptomatic patients after prior revascularization and in order to assess the functional severity of indirect lesions found in coronarography. ${ }^{2}$ In the context of valvular heart defects, SE is used in the functional evaluation of a defect, among others, in patients with low flow-low gradient aortic stenosis (LF-LG AS) (classical and paradoxical). The main features of the study were asymptomatic severe aortic stenosis (AS), secondary mitral regurgitation (MR), severe asymptomatic organic mitral valve regurgitation, and significant asymptomatic mitral stenosis (MVA) $<1.5 \mathrm{~cm}^{2}{ }^{3-5}$

\section{Aim}

The aim of the study covered a detailed analysis of SE tests performed in Poland, including the clinical characteristics of patients, indications for stress diagnostics and applied protocols. Eventually it describes the obtained results in terms of further therapeutic decisions based on a prospective registry covering a complete 2-month study.

\section{Material and methods}

Based on the analysis of the annual retrospective Polish Stress Echocardiography Registry (Pol-STRESS Registry), ${ }^{6}$ large medical institutions including cardiology centers and the majority of universities in Poland were asked to prepare a prospective analysis of data obtained at further diagnosis stages. The tests were performed for 1 month in 2017 by cardiologists or internal medicine practitioners trained in the field of SE. Requirements related to equipment standards and operator's experience have been specified by the Echocardiography Working Group of the Polish Cardiac Society and the appropriate national certification documents.

For a positive SE result in the assessment of myocardial ischemia, the authors agreed on the worsening of contractility in at least 2 segments (in 3 segments for high risk stratification) of the echocardiographic left ventricular model and for the determination of myocardial viability - a typical 2-phase response (improvement of contractility at dobutamine dose up to $20 \mu \mathrm{g} / \mathrm{kg} / \mathrm{min}$ and its deterioration at $40 \mu \mathrm{g} / \mathrm{kg} / \mathrm{min})$. In asymptomatic severe AS, patients qualified for the study were previously diagnosed with aortic valve area (AVA) $<1 \mathrm{~cm}^{2}$ (aortic valve area index (AVAi) $<0.6 \mathrm{~cm}^{2} / \mathrm{m}^{2}$ ) and mean transvalvular gradient $\left(\mathrm{P}_{\text {mean }}\right)>40 \mathrm{~mm} \mathrm{Hg}$ at rest. Poor prognosis featured: AS symptoms, decrease in blood pressure, increase in $\mathrm{P}_{\text {mean }}$ by at least $20 \mathrm{~mm} \mathrm{Hg}$, risk of pulmonary hypertension with systolic pulmonary artery pressure (SPAP) $>60 \mathrm{~mm}$ $\mathrm{Hg}$, and decrease of LVEF. In LF-LG AS (patients with LVEF $<40 \%$, AVA $<1 \mathrm{~cm}^{2}$ and $\mathrm{P}_{\text {mean }}<40 \mathrm{~mm} \mathrm{Hg}$ at rest) the test indicated severe AS when LVEF or stroke volume (SV) improved by more than $20 \%, \mathrm{P}_{\text {mean }}$ elevated $>40 \mathrm{~mm}$ $\mathrm{Hg}$ and AVA remained $<1.0 \mathrm{~cm}^{2}$. The lack of LVEF reserve (stress ejection fraction-rest ejection fraction) was an indication of a poor prognosis. In MR (ischemic etiology), the aim was to assess the EF reserve and the dynamic effective regurgitant orifice (ERO) component with an increase of over $13 \mathrm{~mm}^{2}$. Statistical descriptive data included in the study is presented in numerical and percentage form.

\section{Results}

As many as 17 teaching hospitals and large regional centers in Poland were involved in the study. A total of 189 SE tests were performed, including 178 diagnosing CAD and 11 for VHD assessment (asymptomatic AS, low-flow/ low-gradient AS, MR). Diagnostic tests for CAD were performed in all centers (100\%) and examinations to evaluate VHD varied from 1 center (5\%) for mitral valve disease, to 3 centers (17\%) for both asymptomatic AS and LFLG AS. To evaluate CAD, the protocols used included: DSE (all centers, 100\%), cycling ergometry (1 center, 5\%), treadmill ( 2 centers, $11 \%$ ), and pacing using atrial mode (3 centers, 17\%). In order to evaluate VHD, stress methods were applied in sequence: 1 ) in MR - DSE (1 center, $5 \%$; 2) in LF-LG AS - DSE (2 centers, 11\%) and a cycle ergometer (1 center, $5 \%)$; 3 in patients with asymptomatic AS - DSE (3 centers, $17 \%$ ) or cycle ergometer (1 center, $5 \%$ ).

The study population contained 189 people, including 87 men (46\%) and 102 women (54\%). The average age of the patients was 66.4 years. According to the medical history of the group studied, $63(33 \%)$ people were patients with suspected CAD and 115 (60\%) were those with previously diagnosed CAD based on a history of myocardial infarction (44 people), coronary angiography, and in some patients based on previously performed non-invasive tests. 
A significant VHD had been found in the remaining 14 (7\%) people. Eighty-five (45\%) individuals were subjected to coronary angiography, 58 (30\%) of which were treated with percutaneous coronary angioplasty (PCI) and 16 (8\%) with subsequent coronary artery bypass surgery (CABG). In 2 patients, coronary angiography was used for evaluation for valvular intervention, and in 11 cases it preceded the implantation of an electrotherapy device. Clinically, 31 (17\%) individuals presented symptoms of typical chest angina, 89 (47\%) of atypical angina and 25 (15\%) patients reported non-coronary chest pain (Fig. 1).

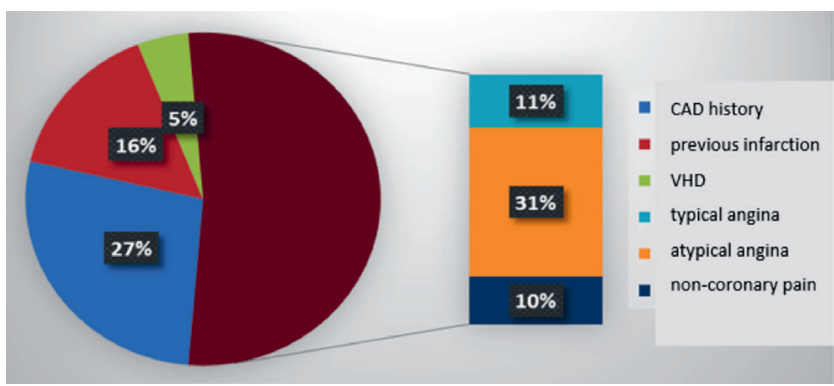

Fig. 1. Initial clinical characteristics of patients

CAD - coronary artery disease; VHD - valvular heart disease.

The analysis of SE studies performed in patients tested for CAD (without previous diagnosis of CAD) revealed that the majority of patients, 45 (71\%), presented intermediate pre-test probability (PTP) of the disease before the test, estimated in accordance with the 2013 European Society of Cardiology (ESC) guidelines. ${ }^{2}$ Low PTP was found in $17(27 \%)$ patients - SE was performed for the verification of ailments and possible functional CAD - and high PTP was described in 1 individual (2\%) - SE was performed for risk stratification and possible revascularization. In all patients analyzed for CAD, 28 (16\%) were assessed for myocardial viability, the remaining 150 (84\%) were subjected to provoking test of possible ischemia. Within the 11 VHD assessment studies, 6 (55\%) involved the analysis of asymptomatic severe AS, 4 studies (36\%) were performed in patients with LF-LG AS and in 1 case (9\%) the study was related to a diagnosis of MR (Fig. 2). The ECG analysis revealed that $166(88 \%)$ patients had regular sinus rhythm at baseline, $13(6.8 \%)$ had atrial fibrillation and 10 (5.2\%) presented pacemaker rhythm. Intraventricular conduction

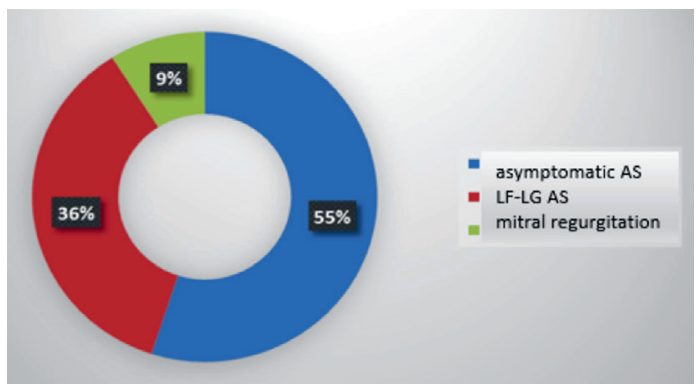

Fig. 2. Valvular heart disease in SE examinations

AS - aortic stenosis; LF-LG AS - low-flow low-gradient aortic stenosis. disorders, being the main element that hindered the interpretation of regular ergometry on the treadmill or bicycle with ECG recording only, included $16(8.5 \%)$ patients with LBBB, 2 (1\%) with RBBB and 16 (8.5\%) cases with nonspecific intraventricular disorders. The LVEF was 55\%, which included LVEF $>50 \%$ in 158 subjects (83\%), 40-50\% in $15(8 \%)$ and less than $40 \%$ in as many as $16(9 \%)$.

Most of the tests $(150,79 \%)$ were carried out in accordance with the recommendations of the Echocardiography Section of the Polish Cardiac Society, ${ }^{1}$ and the remaining 39 (21\%) in accordance with the guidelines of the European Society of Cardiology. ${ }^{4}$ The differences in the 2 protocols are not significant and include, in the first case, early onset of atropine administration (from a dose of $30 \mu \mathrm{g} / \mathrm{kg} / \mathrm{min}$ dobutamine vs $40 \mu \mathrm{g} / \mathrm{kg} / \mathrm{min}$ ), and for the end test criteria, ST-segment elevation $>1 \mathrm{~mm}$ (vs $>2 \mathrm{~mm}$ ) on an ECG. The vast majority of tests, 163 (86\%), were performed by a cardiologist, 20 tests $(10.0 \%)$ by a doctor specializing in cardiology (fellow) and 6 tests (4\%) by an internal medicine doctor. The stress protocol was predominantly with the use of dobutamine $(162,86 \%)$. The remaining tests were performed using a cycle ergometer (12 tests), treadmill (5 tests) or using the stimulation of 10 tests. Dobutamine stress echocardiogram with a full dose of dobutamine ( $40 \mathrm{~g} / \mathrm{kg}$ ) was used in 121 people (75\% of DSE) and $20 \mathrm{~g} / \mathrm{kg}$ in 41 (25\% of DSE) (Fig. 3). Dipyridamole or adenosine were not used in the studies. A positive SE score for the assessment of myocardial ischemia or VHD was obtained in 46 (24\%) people, negative in 110 people (58\%) and non-diagnostic in 33 (18\%). For the assessment of CAD, a positive result was reported in 37 (20\%) patients, negative in 109 (61\%) and non-diagnostic in 32 (19\%). For the evaluation of VHD, a positive result was reported in 9 (82\%) patients, while in negative and non-diagnostic in 1 individual each (9\%) (Fig. 4,5). The average stress time was 14.4 $\mathrm{min}$ (21.6 $\mathrm{min}$ for DSE and $7.3 \mathrm{~min}$ for exercise SE).

The most common reason for terminating the test according to the protocol was obtaining a heart rate limit or applying maximum doses of drugs. This concerned 141 tests. The reasons for the premature termination of the test included: achievement of the test objective, that is, echocardiographically positive result - 39 (20\%); or observed

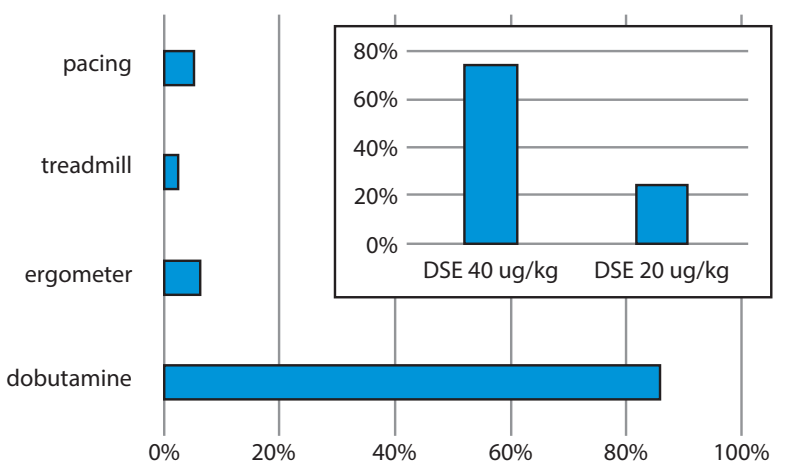

Fig. 3. SE protocols

DSE - dobutamine stress echocardiography. 


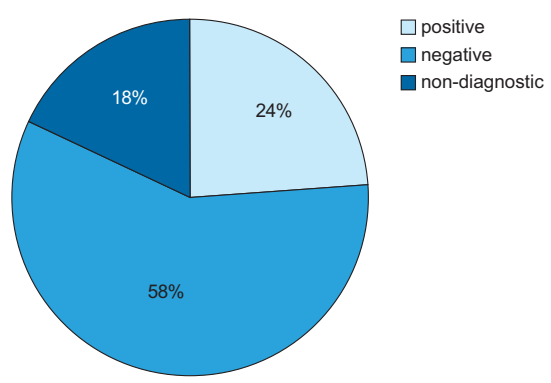

Fig. 4. Results of all SE examinations obtained
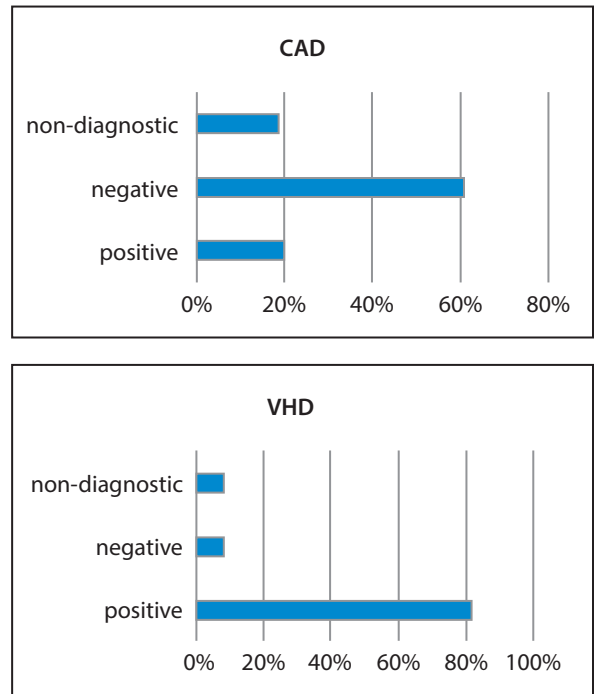

Fig. 5. Results obtained taking into account the diagnostic purpose CAD - coronary artery disease; $\mathrm{VHD}$ - valvular heart disease.

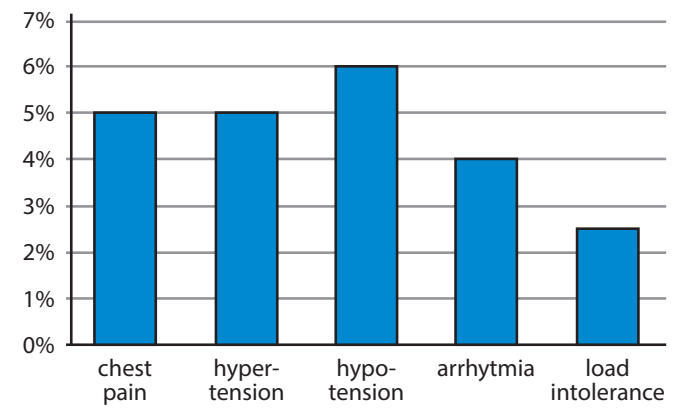

Fig. 6. Adverse effects during SE

adverse reactions - chest pain $9(5 \%)$, excessive increase $(5,2 \%)$ or decrease $(6,3 \%)$ of blood pressure or arrhythmia 8 (4\%). Five (2.5\%) tests were discontinued due to patient's intolerance of the stress agent or $3(1.5 \%)$ at his request (Fig. 6).

The most serious complications of SE (myocardial infarction and death) were not observed. After evaluating the group with CAD and SE performed for the assessment of potential ischaemia, 41 (23\%) patients were referred to coronary angiography; 20 of those had hemodynamically insignificant lesions, 19 people had critical and in $2 \mathrm{pa}$ tients no coronary artery lesions were detected (Fig. 7).

Among 37 patients with positive SE results, 16 patients were diagnosed with critical lesions when subjected

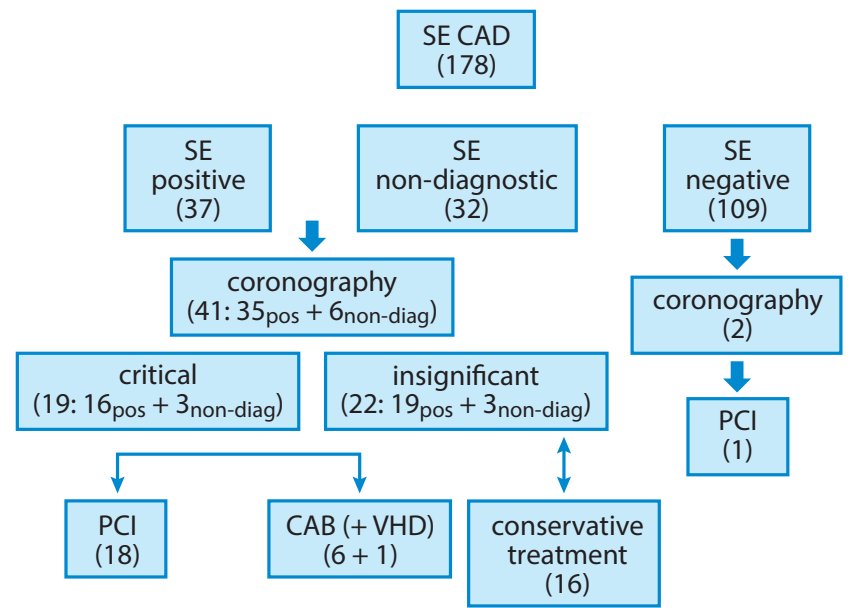

Fig. 7. Diagram of CAD treatment based on SE results obtained (number of patients)

SE - stress echocardiography; CAD - coronary artery disease; VHD - valvular heart disease; $\mathrm{PCl}$ - percutaneous coronary intervention; CABG - coronary artery bypass graft.

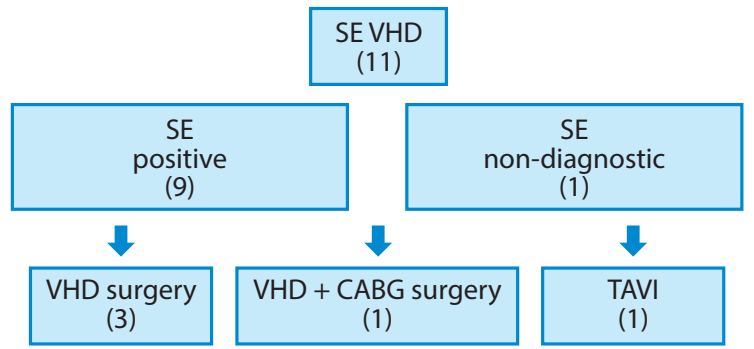

Fig. 8. Diagram of VHD management on the basis of SE results obtained (number of patients)

SE - stress echocardiography; VHD - valvular heart disease;

CABG - coronary artery bypass graft; TAVI - transcutaneous aortic valve implantation.

to coronary angiography. In 2 patients, despite the positive SE result, no angiography was performed. However, among 109 people with negative SE results, 2 patients were subjected to coronary angiography, which revealed in the $1^{\text {st }}$ case insignificant lesions, and in the $2^{\text {nd }}$ critical lesions qualified for PCI were described. The analysis of the significance of the SE results for the decision-making on interventional measurements revealed that 30 patients (from the total study population of 189) were referred for the intervention. That included 19 individuals for percutaneous revascularization ( $44 \%$ of all who underwent angiography and 95\% with significant lesions), 6 (15\%) for surgical revascularization, 3 for valvular surgery, 1 patient qualified for transcutaneous aortic valve implantation (TAVI), and 1 for hybrid CABG and valve surgery (Fig. 7,8).

\section{Discussion}

The study presented shows the results of the first Polish prospective multicenter registry of echocardiographic 
stress tests (PolStress-Echopro). In conjunction with the recently published ${ }^{6}$ retrospective registry, Pol-STRESS is a large database reflecting the current situation and significance of SE in everyday clinical practice in Poland. The analysis involved 17 large cardiology centers, in which a total of 189 SEs were performed. In order to make the registry more objective and reliable, and not limiting its interpretation at the same time, many data points (14.5\%) were rejected in the course of further analysis due to the lack of full access to necessary documentation. In Poland, the most frequently performed stress test is the electrocardiographic exercise test on a treadmill or cycle ergometer, which is mainly due to its wide availability, not the diagnostic power. The sensitivity and specificity of this study are $45 \%$ and $85 \%$, respectively, which, in comparison to SE $(83 \%$ and even $95 \%$ with vasodilator), significantly influences the interpretation of the outcome and subsequently the fate of the patient. ${ }^{2}$ This data in terms of ischemia makes SE the dominant and preferred tool in CAD diagnostics. ${ }^{7}$

An important factor which elucidates accessibility concerns depends on the reimbursements considerations under the healthcare system. Hence, this published registry is considered to raise the awareness among the healthcare community of the increasing role of echocardiographic functional tests, which may encourage them to increase the availability of non-invasive diagnostics measures. All centers performed SE with the use of dobutamine, and only 3 were based on the physiological response of the patient during exercise, although it is known for its higher sensitivity in detecting cardiac ischemia in comparison with dobutamine and dipyrida mole. ${ }^{8}$ Moreover, exercise SE, in addition to providing additional prognostic data on physical performance, can also be used to diagnose patients with valve dysfunction or diastolic insufficiency, which can be frequently co-responsible for a patient's symptoms. ${ }^{9,10} \mathrm{Un}$ fortunately, the limited availability of supine stress ergometers in Poland and the application of relatively cheap provocation agents affects the type of protocol used. Among all institutions included in the study, only 3 used a previously implanted pacemaker. The usage of fast pacing allows the physician to perform the test as accurately and safely as using the previously described modalities, but can influence the result interpretation in the case of ventricular pacing or the occurrence of the Wenckebach point below the target pulse rate. ${ }^{1,10}$ This small number was related to the patient profile and the limited group of patients with a pacemaker. Furthermore, none of the centers used adenosine or dipyridamole. The knowledge of key features and contraindications to a particular type of stress has significant importance in terms of appropriate method selection, making the result even more specific. ${ }^{10}$

The main reason of implementing SE remained CAD and this indication dominated in all centers. Stress echocardiography is recommended in symptomatic patients and in moderate PTP to detect myocardial ischemia, as well as in those without typical angina and LVEF $<50 \%$ or after incomplete revascularization. ${ }^{2}$ This is convergent with our results, where most patients presented intermediate PTP. Only 1 individual was diagnosed with high PTP, which also seems to be reasonable in terms of incident risk and direct qualification for invasive measures. However, Papachristidis et al. ${ }^{11}$ proved that SE can also influence this group of patients. In "only" $41 \%$ of those with high PTP was the SE score positive; $57 \%$ of them were subjected to coronary angiography and $24.6 \%$ were treated with PCI. Compared to the routine approach in accordance with ESC recommendations, a significant reduction in procedure costs was observed, with only a small percentage of serious adverse cardiac and cerebrovascular events (Major Adverse Cardiac and Cerebrovascular Events - MACCE). In current recommendations, SE also plays a key role in more challenging cases of VHD such as: asymptomatic AS, LF-LG AS (both classic low-gradient with $\mathrm{P}_{\text {mean }}<40 \mathrm{~mm} \mathrm{Hg}$ and EOA $<1 \mathrm{~cm}^{2}$, and - paradoxically - with preserved LVEF and low SV $<35 \mathrm{~mL} /$ $\mathrm{m}^{2}$ ), or in MR. Unfortunately, the use of advantages of SE in VHD is limited to only a few centers, which is significantly different from the United Kingdom National Health Service Survey data (almost 99\% of centers), where, except for the VHDs listed in PolStress-Echopro, severe MVA (25\% of centers) and asymptomatic severe aortic regurgitation (18\% of centers) were evaluated. ${ }^{12}$ New guidelines for the treatment of VHDs which appeared recently should influence the wider management of SE in the assessment of VHD, thus improving further therapeutic decisions and patients' prognosis. ${ }^{13,14}$ The guidelines contain some minor modifications related to the outcome of SE performed in asymptomatic AS and in primary asymptomatic MR. ${ }^{13}$ The class IIb $C$ indication for valvular surgery in asymptomatic AS when the mean gradient increased by $>20 \mathrm{~mm}$ $\mathrm{Hg}$ in the SE exercise test, was excluded. Similarly, in patients with primary asymptomatic MR, exertional increase in systolic pressure in the pulmonary artery $>60 \mathrm{~mm} \mathrm{Hg}$ is no longer considered an indication for surgery.

The areas of SE applications are not only limited to CAD and VHD (including valve prostheses) - SE may and should also be performed for other indications such as diastolic dysfunction, cardiomyopathy (dilated, hypertrophic), resynchronization therapy, pulmonary hypertension, congenital heart diseases (coarctation of the aorta, atrial septal defect, tetralogy of Fallot, single-chamber heart), and the athlete's heart. ${ }^{15}$

In the study presented, tests used for CAD assessment revealed the negative SE result in $61 \%$ of patients, which resulted in resignation from invasive testing that could have involved the possibility of serious complications. However, one of the patients with negative DSE who was referred to coronarography had critical coronary lesions.

It should be noted that among the 37 individuals with positive DSE, only 16 had hemodynamically significant lesions. This might be due to inadequate interpretation of the SE outcomes and false-positive results in cases such as: poor test conditions, changes in the basal segment 
of the inferior wall, the presence of asynchrony of the ventricular septum in a patient with LBBB, in a patient after cardiac surgery and in the lesion in the left coronary artery circumflex branch. ${ }^{10}$ As many as $19 \%$ of tests did not have diagnostic power, which corresponds with data from the available literature (5-20\%). ${ }^{16}$ The use of contrast agents would increase the accuracy of diagnosis, but also raise the cost of the study, thus it was not used in any center. ${ }^{17}$

The PolStress-Echopro registry has once again demonstrated the great safety of SE, which, considering its high sensitivity and specificity, makes it a unique diagnostic tool in patients with CAD and VHD. The most frequent stress effects resulted in stenocardial pain (subjective, not related to CAD severity) and fluctuations in blood pressure, which required premature discontinuation of the study. There were no serious adverse events such as death or myocardial infarction observed. For comparison, those complications are described in the UK-NHSS register ( 1 and 8 centers respectively). ${ }^{12}$ Nevertheless, $\mathrm{SE}$ is considered a safe diagnostic method, with mortality rate and myocardial infarction estimated at $<0.01 \%$ and $0.01-0.1 \%$, respectively. ${ }^{18,19}$

An undoubted limitation of this study that needs to be mentioned is the absolute number of the exams analyzed and the time of observation. Both in the retrospective PolSTRESS Registry and the currently referenced prospective PolStress-Echopro, the number of SEs performed for the purpose of CAD assessment was predominant. This data is consistent and should be interpreted in a coherent and comprehensive manner, as well to confirm the conclusions of the work presented below on the necessity of wider dissemination of this method in the assessment of VHD. However, when analyzing the number of tests and the time of observation between both registries, the results obtained are proportional to the time of observation.

\section{Conclusions}

The most commonly used echocardiographic stress test in the diagnosis of coronary heart disease is DSE. Negative test results in the CAD diagnoses made it possible to avoid invasive coronary angiography in almost half of the patients. The proven high value and SE safety in assessing the significance of selected heart valve defects should lead to more frequent use of these methods in the qualification of patients for surgical treatment.

\section{ORCID iDs}

Zbigniew Tadeusz Gąsior (1) https://orcid.org/0000-0003-3616-8932 Beata Zaborska (1) https://orcid.org/0000-0001-5513-8371 Katarzyna Mizia-Stec (1) https://orcid.org/0000-0001-6907-2799 Piotr Gościniak (1) https://orcid.org/0000-0003-1290-0391 Marta Marcinkiewicz-Siemion (1) https://orcid.org/0000-0002-6531-2705 Barbara Brzezińska (10) https://orcid.org/0000-0002-5350-1568 Tomasz Gąsior (1) https://orcid.org/0000-0001-5088-4164 Agnieszka Olszanecka (1D) https://orcid.org/0000-0001-7020-9641 Edyta Płońska-Gościniak (1) https://orcid.org/0000-0003-1632-3261

\section{References}

1. Płońska-Gościniak E, Gackowski A, Gạsior Z, et al.; Echocardiography Working Group of the Polish Cardiac Society. Recommendations of the Echocardiography Working Group of the Polish Cardiac Society for stress echocardiography use in clinical practice 2011 [in Polish]. Kardiol Pol. 2011;69(6):642-648.

2. Montalescot G, Sechtem U, Achenbach S, et al. ESC guidelines on the management of stable coronary artery disease: The Task Force on the management of stable coronary artery disease of the European Society of Cardiology. Eur Heart J. 2013;34(38):2949-3003.

3. Lancellotti P, Płońska-Gościniak E, Garbi M, et al. Cardiovascular imaging practice in Europe: A report from the European Association of Cardiovascular Imaging. Eur Heart J Cardiovasc Imaging. 2015;16(7):697-702.

4. Sicari R, Nihoyannopoulos P, Evangelista A, et al; European Association of Echocardiography. Stress echocardiography expert consensus statement: European Association of Echocardiography (EAE) (a registered branch of the ESC). Eur J Echocardiogr. 2008;9(4):415-437.

5. Pellikka PA, Nagueh SF, Elhendy AA, KuehI CA, Sawada SG; American Society of Echocardiography. American Society of Echocardiography Recommendations for Performance, Interpretation, and Application of Stress Echocardiography. J Am Soc Echocardiogr. 2007;7(9):1021-1041.

6. Płońska-Gościniak E, Kasprzak JD, Olędzki S, et al. Polish Stress Echocardiography Registry (Pol-STRESS Registry): A multicenter study. Stress echo in Poland: Numbers, settings, results and complications. Kardiol Pol. 2017;75(9):922-930.

7. Kolh P, Windecker S, Alfonso F, et al. 2014 ESC/EACTS Guidelines on myocardial revascularization: The Task Force on Myocardial Revascularization of the European Society of Cardiology (ESC) and the European Association for Cardio-Thoracic Surgery (EACTS). Developed with the special contribution of the European Association of Percutaneous Cardiovascular Interventions (EAPCI). Eur J Cardiothorac Surg. 2014;46(4):517-592.

8. Grewal J, McCully RB, Kane GC, Lam C, Pellikka PA. Left ventricular function and exercise capacity. JAMA. 2009;301(3):286-294.

9. Picano E, Pingitore A, Conti U, et al. Enhanced sensitivity for detection of coronary artery disease by addition of atropine to dipyridamole echocardiography. Eur Heart J. 1993;14(9):1216-1222.

10. Lipiec P, Bąk J, Braksator W, et al. Transthoracic echocardiography in adults - guidelines of the Working Group on Echocardiography of the Polish Cardiac Society [in Polish]. Kardiol Pol. 2018;76(2):488-493.

11. Papachristidis A, Demarco DC, RoperD, etal. The safety, effcacy and costeffectiveness of stress echocardiography in patients with high pretest probability of coronary artery disease. Open Heart. 2017;4(2):e000605.

12. Bhattacharyya S, Chehab O, Khattar R, Lloyd G, Senior R; British Society of Echocardiography. Stress echocardiography in clinical practice: A United Kingdom National Health Service Survey on behalf of the British Society of Echocardiography. Eur Heart J Cardiovasc Imaging. 2014;15(2):158-163.

13. Baumgartner H, Falk V, Bax JJ, et al; ESC Scientific Document Group. 2017 ESC/EACTS Guidelines for the management of valvular heart disease. Eur Heart J. 2017;38(36):2739-2791.

14. Gentry lii JL, Phelan D, Desai MY, Griffin BP. The role of stress echocardiography in valvular heart disease: A current appraisal. Cardiology. 2017;137(3):137-150.

15. Lancellotti P, Pellikka PA, Budts W, et al. The clinical use of stress echocardiography in non-ischaemic heart disease: Recommendations from the European Association of Cardiovascular Imaging and the American Society of Echocardiography. Eur Heart J Cardiovasc Imaging. 2016;17(11):1191-1229.

16. Shah BN, Balaji G, Alhajiri A, Ramzy IS, Ahmadvazir S, Senior R. Incremental diagnostic and prognostic value of contemporary stress echocardiography in a chest pain unit: Mortality and morbidity outcomes from a real-world setting. Circ Cardiovasc Imaging. 2013;6(2): 202-209.

17. Senior R, Becher $\mathrm{H}$, Monaghan $\mathrm{M}$, et al. Contrast echocardiography: Evidence-based recommendations by European Association of Echocardiography. Eur J Echocardiogr. 2009;10(2):194-212.

18. Geleijnse ML, Krenning BJ, Nemes A, et al. Incidence, pathophysiology, and treatment of complications during dobutamine-atropine stress echocardiography. Circulation. 2010;121(15):1756-1767.

19. Weidmann B, Lepique CU, Jansen W, Stoiber WU, Tauchert MO. Myocardial infarction as a complication of dobutamine stress echocardiography. J Am Soc Echocardiogr. 1997;10(7):768-771. 